\title{
Demonstration of calcification fronts by in vivo and in vitro tetracycline labelling
}

\author{
JOHN MCCLURE \\ From the Adelaide Bone and Joint Research Unit, Division of Tissue Pathology, Institute of Medical and Veterinary \\ Science, Frome Road, Adelaide, South Australia 5000
}

SUMMARY In patients with chronic renal failure estimations of the extent of calcification fronts delineated by in vivo tetracycline labelling correlated well with estimates obtained after staining the fronts with haematoxylin dye (Raina method). Similarly there was a good correlation between the Raina method and the demonstration of calcification fronts with ultraviolet (UV) light fluorescence after immersion of the bone tissue in fixative containing a freshly prepared solution of $1 \%$ tetracycline. These studies indicate that this latter is a valid and useful technique for demonstrating calcification fronts in a situation where tetracycline cannot be administered systemically.

The site of initial mineralisation of lamellar bone is at the junction between mineralised bone and osteoid. This area is usually referred to as the calcification front. The demonstration and quantification of the calcification front is important in distinguishing conditions characterised by an increased volume of osteoid. Estimation of the percentage of an osteoid seam bearing a calcification front may be readily performed by a line-intersect technique using an eyepiece graticule. To do this depends on delineating satisfactorily the calcification front in undecalcified thin bone sections and on easily distinguishing osteoid from mineralised tissue.

The calcification front is evidently a complex structure and may be demonstrated by several quite different but apparently specific staining methods which include Sudan black, cobalt salts, toluidine blue or solochrome cyanine $\mathbf{R}^{1}$. Sudan black stains lipid ${ }^{2}$, cobalt salts stain phosphate ${ }^{3}$ and toluidine blue by exhibiting metachromasia indicates the presence of a mucopolysaccharide. Raina ${ }^{4}$ has reported that in haematoxylin and eosin stained undecalcified sections the calcification front appears as a line of basophilic granular material between the pink staining osteoid tissue and the underlying calcified bone (Raina method). Calcification fronts may also be demonstrated by administering an in vivo tetracycline label and studying undecalcified sections by fluorescence microscopy ${ }^{5}$. Since not all laboratories have been successful in the application of some of the above mentioned staining methods, the generally accepted method of demonstrating calcification fronts is tetracycline labelling.

Accepted for publication 12 May 1982
Rasmussen and Bordier ${ }^{6}$ have studied the correlation between quantitative estimations of calcification fronts demonstrated by in vivo tetracycline labelling on the one hand and either toluidine blue staining, Sudan black staining or cobalt salt staining on the other and found hight degrees of correlation for the three staining methods These findings were valid not only for normal subjects, but also for patients with nutritional osteomalacia before and after vitamin D therapy. A similar correlative study has not been performed for the Raina method and the present report describes such a study.

In addition, Aaron ${ }^{7}$ has reported that the immersion of a bone sample in a solution of tetracycline prior to the preparation of undecalcified sections results in a satisfactory delineation of the calcification fronts. Therefore, bone biopsies have been taken from patients without prior in vivo labelling and fixed in a buffered solution of formalin containing $1 \%$ tetracycline to determine if calcification fronts could be labelled by this in vitro approach and to perform a correlative study with the Raina method.

\section{Material and methods}

Bone biopsies from two groups of 14 patients were studied. All patients were in chronic renal failure for varying causes and were on intermittent haemodialysis. The patients in the first group were given doxycycline 100 mg daily for three days ending two weeks before bone biopsy. In all cases a bone biopsy was taken (with informed consent) from the left anterior iliac crest. The bone cores were taken just behind the anterior spine and through the top of the crest passing down between outer 
and inner cortices. The biopsies from the first group of patients (who had received doxycycline in vivo) were fixed in $10 \%$ buffered formalin for a minimum of $48 \mathrm{~h}$. Material from the second group was fixed in $10 \%$ buffered formalin containing a $1 \%$ solution of tetracycline prepared immediately before use. The fixation period was again a minimum of $48 \mathrm{~h}$.

After fixation all biopsies were processed in an identical manner initially being dehydrated through graded alcohols, cleared with acetone and placed in a $1: 1$ acetone: Araldite solution. Blocks were prepared by setting the bone material in Araldite $\mathrm{D}$ epoxy solution in rubber moulds in an oven at $56^{\circ} \mathrm{C}$. Sections were cut from the blocks at a thickness of $7 \mu \mathrm{m}$ using a Jung $\mathrm{K}$ microtome. Multiple sections were cut and mounted on gelatinised slides and dried in a hot oven overnight. Araldite was removed with an alcohol/potassium hydroxide solution. Alternate slides were stained by the haematoxylin and cosin (HE) method and the others coverslipped with DPX mountant without staining for examination in UV light.

For the quantitative estimation of the extent of calcification front in both $\mathrm{HE}$ and fluorescent preparations an eyepiece graticule containing 21 grid lines was used. Fields were examined at an optical magnification of $\times 150$. Fields were chosen at random throughout the available slides until a total of 100 had been examined in each case. The number of grid-line intersections with an osteoid covered trabecular surface with and without calcification fronts was counted and the percentage of the osteoid surface bearing a calcification front was calculated.

The estimations of calcification front extents using HE staining and UV light fluorescence were made separately without knowledge of either result by independent operators. For each group the estimates obtained by each method were analysed. The mean value and variance were calculated together with Pearson's correlation coefficient and an $X Y$ plot drawn to illustrate the relationship. A Wilcoxon signed rank test was also applied.

\section{Results}

Satisfactory demonstration of calcification fronts was obtained in HE-stained sections. As reported by Raina ${ }^{+}$ the calcification front appeared as a basophilic line between the pink-staining osteoid tissue and the underlying blue-staining calcified tissues (Fig. 1). Fluorescence of the calcification fronts was observed in both the cases labelled in vivo (Fig. 2) and those which had been fixed in a solution of $1 \%$ tetracycline (Figs. 3 and 4).

The analysis of the comparison between the results obtained by tetracycline labelling in vivo and HE staining is given in Table 1 and the corresponding XY plot is illustrated in Fig. 5. Similarly the results obtained after tetracycline labelling in vitro and HE staining are given in

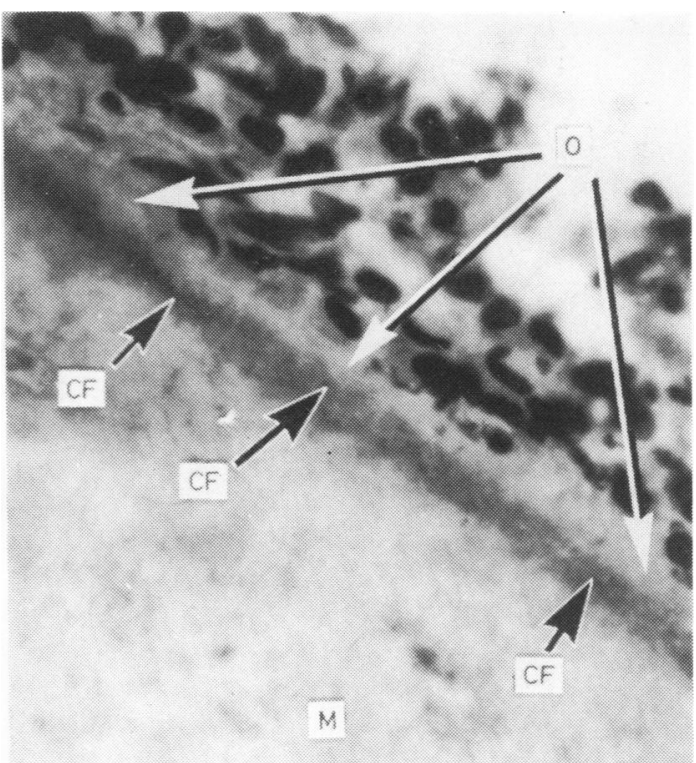

Fig. I The calcification fromt (CF) lies between the osteoid $(O)$ and mineralised tissue $(M)$. Haematorvlin and eosin $\times 400$ ).

Table 2 and illustrated in Fig. 6. $\mathrm{R}$ is Pearson's correlation coefficient and the $Z$ value is the result of the application of the Wilcoxon signed rank test. If this $Z$ value is greater than 1.7 then there is a significant difference between the results obtained by the two methods $(p<0.05)$.

The calcification front extents of cases included in this study showed a wide range of values, although cases were not specifically chosen to obtain this effect. The calcification front extent should normally be greater than $60 \%$ and figures below this value associated with an increased osteoid volume and thickened osteoid seams are indicative of osteomalacia ${ }^{8}$. In the in vivo component six cases had a value greater than and eight cases a value less than $60 \%$. In the in vitro component seven cases each had values either above or below $60 \%$. The maximum number of birefringent osteoid lamellae seen in polarised light was counted for each specimen and the mean and standard error of the mean (SEM) calculated for both in vivo and in vitro study groups and for those cases within each group with a calcification front extent either greater or less than $60 \%$. In the in vivo study group the group mean was $2 \cdot 8$ $(\mathrm{SEM}=0 \cdot 3)$, for cases $>60 \%$ it was $2 \cdot 0(\mathrm{SEM}=0 \cdot 4)$ and for cases $<60 \%$ it was $3.4(\mathrm{SEM}=0.4)$. In the in vitro study group the group mean was $3 \cdot 4($ SEM $=0 \cdot 4)$. for cases $>60 \%$ it was $2 \cdot 2(\mathrm{SEM}=0.4)$ and for cases $<60 \%$ it was $4 \cdot 7(\mathrm{SEM}=0 \cdot 2)$.

\section{Discussion}

The results obtained confirm Raina's observation that calcification fronts may be demonstrated in undecalcified 


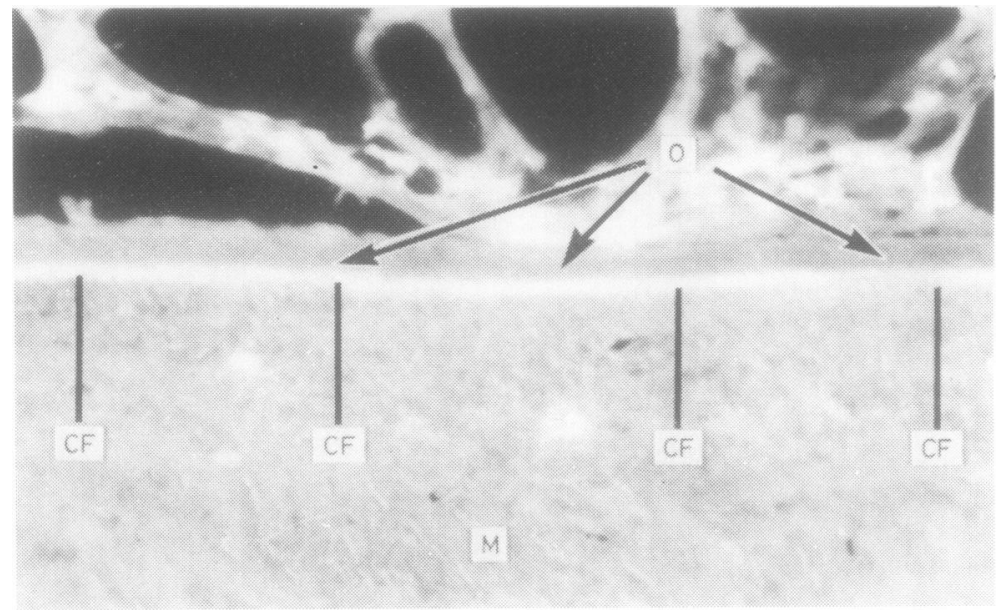

Fig. 2 Calcification front (CF) labelled by administration of tetracycline in vivo. $O$ is osteoid and $M$ is mineralised tissue. UV light fluorescence $\times 200$.

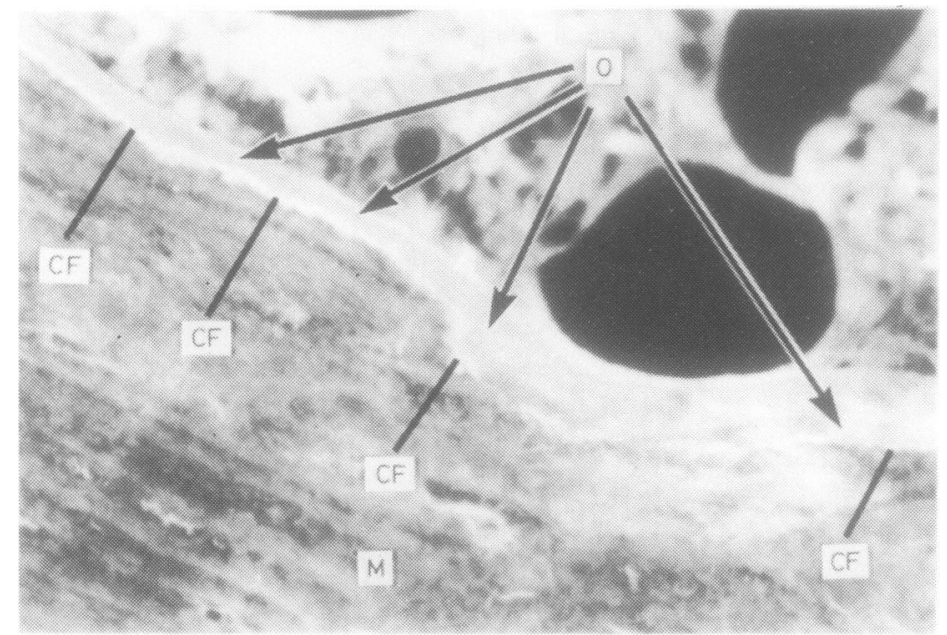

Fig. 3 Calcification from (CF) labelled by immersion of bone biopsy in fixative containing a $1 \%$ solution of tetracvcline. $O$ is osteoid and $M$ is mineralised tissue. UV light $\supset$ fluorescence $\times 200$.

sections by haematoxylin and eosin staining. Quantitative estimations of the extent of calcification fronts defined in this fashion do not differ significantly from estimations performed on fronts demonstrated by UV light fluorescence after in vivo tetracycline labelling in the same patients. In fact, there is a high degree of correlation. It is evident that the calcification front reacts with haematoxylin dve and that the reactive component is closely related. to that which chelates with tetracycline administered as an in vivo label.

The second and probably more important aspect of this study is that the immersion of the bone biopsy in buffered fixative containing a freshly prepared $1 \%$ solution of tetracycline is followed by incorporation of the tetracycline into the calcification front where it may be visualised by UV light immunofluorescence. The estimations of the extent of this reaction correlate to a high degree with estimations of the calcification front delineated by haematoxylin dye and since these in turn correlate with the tetracycline in vivo labelling technique then the fluorescence obtained after immersion in a fixative solution containing tetracycline is a valid method of demonstrating the calcification front. That this method is successful indicates that the process whereby tetracycline reaches the calcification front is a passive one, presumably depending upon diffusion and not requiring cellular passage.

The extent of the calcification front was estimated as the percentage of the total osteoid surface bearing such a front. A decrease is said to be indicative of a defect in bone mineralisation ${ }^{6}$. In some conditions such as primary hyperparathyroidism and Paget's disease of bone there is an increase in the thickness of active osteoid seams and an increase in the extent of the trabecular surface covered by 


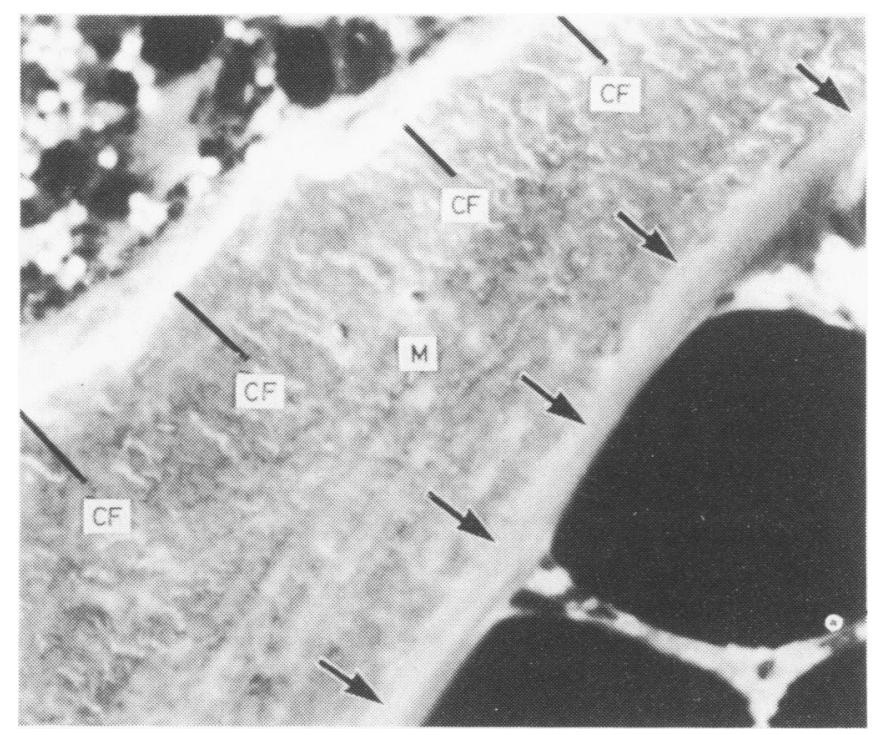

Fig. 4 Calcification fromt (CF) labelled by tetracycline in vitro. The calcification fiont in this zone is broad. Note the absence of calcific ation fromt on contralateral side at the junction of osteoid and mineralized tissuc (arrowheads). UV light fluorescence $\times 200$.

Table 1 Results of the comparison of calcification front values obtained after tetracycline label (in vivo) with those obtained after HE staining

\begin{tabular}{lllll}
\hline Method & Mean & Variance & $R$ & $Z$ \\
\hline Tetracycline label (in vivo) & 50.54 & 466.87 & 0.9534 & 0.4544 \\
Haematoxylin and eosin & $51 \cdot 11$ & 362.84 & & \\
\hline
\end{tabular}

$\mathrm{R}=$ Pearson's correlation coefficient

$\mathrm{Z}=$ Value obtained in Wilcoxon signed rank test.

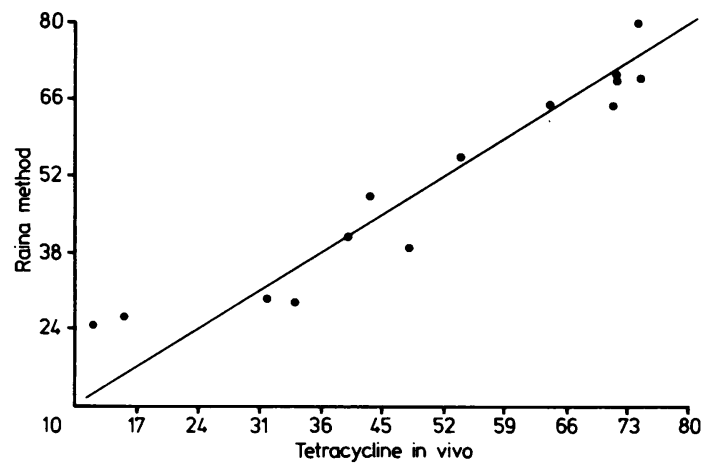

Fig. $5 X Y$ plot of $C F$ values obtained by tetracycline given in vivo versus values obtained by the Raina method

Table 2 Results of the comparison of calcification front values obtained after fixation in a $1 \%$ solution of tetracycline with values obtained after $H E$ staining

\begin{tabular}{lllll}
\hline Method & Mean & Variance & $R$ & $Z$ \\
\hline Tetracycline label (in vitro) & $61 \cdot 32$ & $406 \cdot 38$ & 0.9386 & $-0 \cdot 1570$ \\
Haematuxylin and eosin & $61 \cdot 29$ & $402 \cdot 65$ & & \\
\hline
\end{tabular}

For $\mathrm{R}$ and $\mathrm{Z}$. see Table 1 .

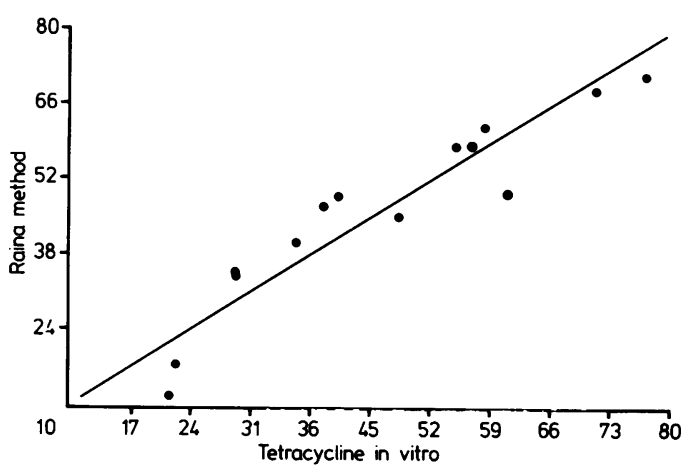

Fig. 6 XY plot of CF values obtained by tetracycline labelling in vitro versus values obtained by the Raina method.

osteoid, but the percentage of the osteoid surface bearing a calcification front is normal.

In these situations there is a delay in either the onset or achievement of mineralisation. This contrasts with the situation in vitamin D deficiency (osteomalacia) in which the osteoid volume is also increased, but the percentage of the osteoid surface bearing a calcification front is reduced due to a defect in the initiation of mineralisation.

This laboratory deals with a large number of bone biopsies from patients with chronic renal failure and it is the experience that oral administration of tetracycline is not inevitably followed by labelling of the calcification fronts in these patients presumably due to variation in absorption of the tetracycline. The success of the in vitro technique described here offers a simple and reproducible technique for tetracycline labelling of calcification fronts and is likely to be of particular use in situations where 
systemic administration of tetracycline is difficult or impossible.

Great difficulty has been experienced in this laboratory in the use of toluidine blue for the demonstration of calcification fronts and, in contrast, the Raina method has been found to be useful, although it is not commonly used by workers in this field. The in vivo component of the present study validates the Raina method as a method of demonstrating calcification fronts.

The majority of undecalcified bone biopsies studied in this laboratory is from chronic renal failure patients and it was not. therefore. possible to include in the study material from patients with metabolic bone disease of other causes. However, the material studied included cases ranging from those with evident osteomalacia (reduced calcification fronts and thickened osteoid seams) to those with no histoquantitative abnormality. Therefore. the methods described are valid for the demonstration of calcification front extents across the range from low abnormal to high normal values.
I wish to thank Mrs $M$ Rowlands for typing the manuscript.

\section{References}

' Matrajt H. Hioco D. Solechrome cranin R as an indicator de of hone morphology. Stain Techmol 1966:41:97-100.

- Irving JT. The sudanophil material at sites of calcilication. Arch ()ral Biol 1963:8:735-45

"Burstone MS. Calcification in biological msems. Washington

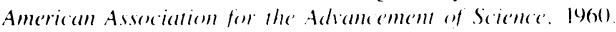

${ }^{4}$ Raina V. Normal osteoid tisuce. I (lin Pathol 1972:25:224) 32.

'Frost HM. Tetracycline-based histolegical analysin of hone remodelling. Calcif Tissue Res 1969:3:211 37

"Rasmussen H. Bordier P. The physiological and cellular hasis of metabolic bone disease. Baltimore: Williams and Wilkins. 1974

Aaron JE. Histological aspects of the relationship between sitamin D and bone. In: Lawson DEM. ed Vitamin I) London: Academic Pres. 1978 .

* Melvin KEW. Hepner (iW: Bordier P. Neale (i. Joplin (iF. Calcium metabolism and hone patholenge in adult cocliat disease. () I Med 197():39:83 113

Requests for reprints to: Dr J McClure. Institute of Medical and Veterinary Science, Box 14, Rundle Street Post Office. Adelaide. South Australia 5000 